\title{
FILOSOFÍA DE LAS MATEMÁTICAS, TEORÍA DE CARDINALES GRANDES Y SUS BASES COGNITIVAS*
}

\author{
Wilfredo Quezada \\ Universidad de Santiago de Chile \\ wilfredo.quezada@usach.cl
}

\begin{abstract}
RESUMEN / ABSTRACT
En este artículo se examinan algunas implicaciones del naturalismo matemático de P. Maddy como una concepción filosófica que permite superar las dificultades del ficcionalismo y el realismo fisicalista en matemáticas. Aparte de esto, la mayor virtud de tal concepción parece ser que resuelve el problema que plantea para la aplicabilidad de la matemática el no asumir la tesis de indispensabilidad de Quine sin comprometerse con su holismo confirmacional. A continuación, sobre la base de dificultades intrínsecas al programa de Maddy, exploramos un camino naturalista mejor motivado, el enfoque cognitivista corporeizado, y sugerimos que él permite explicar de manera adecuada la postulación de ciertos cardinales grandes, en particular, la aceptación de conjuntos no-construibles.

PAlabras Clave: naturalismo matemático, teoría de conjuntos, cardinales grandes, conjunto construible, cognición corporeizada.
\end{abstract}

\section{Philosophy of Mathematics AND the COGNITIVE BASIS OF THE LARGE CARDINALS THEORY}

In this paper we examine in first place some philosophical implications of P. Maddy's mathematical naturalism as a philosophical account which allows us to overcome the drawbacks associated to both fictionalism and physicalistic realism in philosophy of mathematics. Besides that, the main virtue of that account seems to lie in the solution it provides to the applicability of mathematics problem when rejecting both the indispensability thesis and confirmative holism postulated by Quine. In second place, on the basis of difficulties intrinsic to Maddy's philosophical program, we explore a better motivated naturalistic option -the embodied cognition approach-and suggest that it allows to adequately explain why most of set-theory specialists use to postulate the existence of some large cardinals, in particular, non-constructible sets.

KEYWORDS: mathematical naturalism, set theory, large cardinals, constructible set, embodied cognition.

* Este artículo se realizó en el contexto del Proyecto Fondecyt Regular № 1080415. 


\section{Introducción}

RRA El actual debate en filosofía de las matemáticas, si bien relacionado con las tradicionales discusiones sobre la realidad o irrealidad de las entidades matemáticas y el problema de la verdad de los enunciados matemáticos que nos legaran Frege, Hilbert, Brouwer y Gödel, parece dominado ahora por tres cuestiones particulares: cuestiones semánticas (problemas de referencia y de verdad matemática), cuestiones epistemológicas (naturaleza del conocimiento matemático) y cuestiones relacionadas con la aplicabilidad de la matemática. Esta agenda ha sido provista básicamente por el trabajo de W. Quine (1951, 1955 y 1969), H. Putnam (1975) y P. Benacerraf(1973). De las tres cuestiones mencionadas, la atingente a la aplicabilidad parece ser, sin embargo, aquella que suscita la mayor atención. Su origen es una sugerencia relativamente simple defendida tanto por Quine como por Putnam. Ellos sugirieron que la cuestión sobre la realidad de los entes matemáticos depende de que se explique por qué ellos parecen ser tan fundamentales a la hora de hacer ciencia fáctica. En este artículo exploraremos algunas de las consecuencias que se siguen de no aceptar tal respuesta realista y, por otro lado, asumir una base de carácter naturalista como la sugerida por Quine para ofrecer una respuesta alternativa, aunque sobre bases muy diferentes a las quineanas.

\section{La tesis de indispensabilidad y la naturalización de la matemática}

Ya que, según los anteriores autores, no tenemos mejor explicación para la singular aplicabilidad de la matemática que decir que simplemente sin las entidades matemáticas la empresa científica exitosa sería imposible, parece necesario admitirlas como "indispensables" para dicha empresa, al menos tanto como lo son las entidades teóricas (no matemáticas) que suelen admitir otros científicos, en particular, los físicos. En otras palabras, esta respuesta nos obliga a admitir su simple realidad. Ésta ha llegado a ser conocida entonces como la "tesis de la indispensabilidad" (Colyvan 2001 y Resnik 1997) y se reconoce como el mejor argumento contemporáneo a favor de lo que podríamos llamar un realismo o platonismo fisicalista de las entidades matemáticas (Maddy 1990 y 1997)). Un intento posterior de enfrentar las dos cuestiones señaladas al principio, y en particular el argumento de indispensabilidad, es la filosofía ficcionalista de las matemáticas desarrollada a partir de los ochenta por H. Field y otros (Field 1980 y 1989; Wagner 1982). De acuerdo con Field, la única forma de enfrentar directamente dicho argumento es asumir algo obvio: que, evaluados semánticamente de acuerdo a su contenido literal, los enunciados de la matemática pura son literalmente falsos (Field 1989 , pp. 2ss.). En otras palabras, ellos presuponen un compromiso con entidades abstractas - conjuntos de Borel, números complejos, hiper-reales, espacios de Hilbert, etc.- que, de hecho, no existen. Aunque, por tanto, la matemática es literalmente falsa, los ficcionalistas sostienen que sus enunciados podrían ser verdaderos en el sentido o en la medida en que decimos que enunciados de ficción lo son: solo que en tal caso arriesgamos admitir que " $2+2=4$ " es solo verdadera en relación con una historia, tanto 
como lo es "Sancho Panza era el escudero de Don Quijote" en relación con otra (Field 1989, p. 5).

Por otro lado, a diferencia de enunciados de simple ficción, los enunciados matemáticos son instrumentos extraordinariamente útiles en dominios fuera de la matemática. Los ficcionalistas están conscientes de que esta última afirmación no es suficiente para bloquear el argumento de indispensabilidad, por lo que avanzan una tesis tan atrevida como la primera: ellos intentan mostrar (basados en un resultado de "conservatividad" aplicable a toda teoría formal) que las entidades matemáticas son formalmente eliminables del discurso científico, en particular, del discurso de la física. Esto es lo que ellos llaman el proyecto de "nominalización de la física" (Field 1980). Como se ve, estas tesis contestan directamente las cuestiones de la verdad y de la aplicabilidad aunque no contestan la cuestión del conocimiento. En este sentido, los ficcionalistas se ven llevados a afirmar que el conocimiento matemático es fundamentalmente conocimiento lógico de carácter modal, en palabras simples, conocimiento acerca de consistencia lógica.

El ficcionalismo, así, parece plantear desafíos importantes y originales. En primer lugar, ¿es la falsedad de la matemática la única opción semántica disponible si se desea evitar el realismo de las entidades matemáticas? En segundo lugar, ¿es la conservatividad de la matemática un criterio seguro para deshacerse de ella en la ciencia fáctica y abrazar un simple instrumentalismo matemático? En tercer lugar, ¿cuál es el estatus del conocimiento lógico modal y sus implicaciones para el contenido de la matemática? Como hemos argumentado en otro lugar (Quezada 2004a), las dos primeras cuestiones deben ser contestadas negativamente y la última depende de contrastar el proyecto ficcionalista con un proyecto filosófico estructuralmente semejante, la concepción logicista desarrollada por Russell entre 1903 y 1907. Esta concepción sostiene, en forma simplificada, que la matemática es la clase de todas las oraciones de la forma 'si p entonces q', donde 'p' puede ser un axioma de una teoría particular y ' $\mathrm{q}$ ' un teorema de esa teoría, lo que ha hecho conocida a esta visión como si-entoncismo (B. Russell 1903 y 1973). En nuestra opinión, el si-entoncismo russelliano puede interpretarse desde el punto de vista del conocimiento matemático como un ficcionalismo limitado, pues lleva a sostener, al igual que este último, que dicho conocimiento es conocimiento lógico modal (Quezada 2004b y Putnam 1975). Sin embargo, una caracterización detallada del conocimiento lógico modal envuelto en ambas concepciones muestra que ellas se verán inevitablemente comprometidas con una dosis no menor de realismo, en la medida en que deben asumir la existencia de modelos posibles, lo que debilita directamente su motivación antirrealista inicial (W. Quezada 2005b). Lo dicho anteriormente permite, finalmente, caracterizar el programa ficcionalista y el si-entoncista como programas de base logicista.

Por otro lado, el mismo Quine ha dado un ímpetu especial a una idea radicalmente opuesta a tales programas y que ha gobernado paralelamente la actual reflexión filosófica sobre la matemática, la idea de 'naturalizar' el conocimiento matemático, es decir, de insistir que sus reglas de aceptación y justificación deberían ser básicamente las mismas que las del resto de las ciencias (Quine 1981). Esta idea, más allá de las propias concepciones de Quine sobre las entidades matemáticas, sugiere un camino muy 
distinto para responder las cuestiones semánticas, epistemológicas y de aplicabilidad vinculadas a la matemática. En una visión naturalista, la matemática no goza de una objetividad distinta o superior a otras ciencias, se somete a sus métodos, y por lo tanto es tan falible y corregible como aquéllas. De esto se sigue que los problemas sobre la verdad y el conocimiento matemáticos no pueden ser esencialmente diferentes a aquellos que enfrentan el resto de las ciencias.

Varios han sido los filósofos que han asumido la tarea de dar más contenido a la idea de naturalizar la matemática, por ejemplo, P. Maddy y P. Kitcher. Maddy, basada en una orientación wittgensteiniana de la filosofía de la matemática, ha propuesto un naturalismo pragmático, de acuerdo al cual las decisiones prácticas de los matemáticos son el tribunal final para la evaluación de la verdad de los enunciados matemáticos (Maddy 1997).

Kitcher, por otro lado (Kitcher 1984) ha defendido un naturalismo evolucionista basado en las operaciones realizadas por un sujeto ideal. Cada uno de estos programas naturalistas ofrece respuestas relativamente persuasivas al argumento de la indispensabilidad, evitando simultáneamente un compromiso con entidades abstractas y, junto con ello, iluminando de manera interesante cuestiones vinculadas a la práctica y al cambio conceptual en matemáticas, que el ficcionalismo no considera. Sin embargo, estas visiones pueden enfrentar problemas. Tal vez el más presionante es el que se deriva para estas concepciones de su compromiso con el empirismo de Quine, que no parece alejarse demasiado del empirismo de Mill (Alemán 2001 y Shapiro 1997). Aunque esta crítica puede ser superada apelando a las características diferenciadoras del empirismo quineano, dicha apelación llevaría derechamente al compromiso con una tesis epistemológicamente compleja que se sigue de las tesis naturalizadas de Quine, el holismo naturalista ( $\mathrm{HN}$ de aquí en adelante): toda evidencia confirmando a otras disciplinas o ciencias naturales es evidencia confirmando la matemática y, por tanto, nada que se acepte en tales ciencias es epistémicamente inofensivo para la matemática y viceversa. Esto permite concluir, a su vez, que los métodos de la matemática y sus criterios de aceptación son interdependientes del resto de las ciencias, lo que condiciona la práctica matemática inmediatamente. Maddy, en reacción a esta conclusión, propone un camino radical $^{1}$ : rechazar HN manteniendo el naturalismo y, por tanto, abandonando el argumento de indispensabilidad. Su argumentación se focaliza fundamentalmente en la práctica de la teoría de conjuntos y, en particular, en la teoría de cardinales grandes. Según Maddy, el holismo quineano simplemente no se ajusta de ninguna manera a los métodos que los matemáticos realmente usan. Por ejemplo, el $\mathrm{HN}$ requeriría que la metodología que rige la práctica de la matemática (el espacio en el cual se determinan cuestiones de aceptación y rechazo de hipótesis o teorías en aquella) tome en cuenta desarrollos en la aplicación de la matemática a la física. En particular, los matemáticos trabajando en teoría de conjuntos con cardinales grandes o muy grandes e intentando resolver cuestiones relativas a las implicaciones de aceptar el axioma de constructibilidad 
$(V=L)^{2}$ o el axioma que afirma la existencia de cardinales medibles $(\mathrm{CM})^{3}$ deberían tomar en cuenta la aplicación de la matemática del continuo en la física cuántica, ya que en ésta rechazamos el supuesto de un continuo material. Pero, como cualquiera podría comprobar, los matemáticos que hacen teoría de conjuntos no muestran un interés mayor en tales desarrollos que el que puede mostrar cualquier observador neutral. En contraste al holismo naturalista, Maddy favorece, por tanto, una concepción metodológica radical, que, aunque acepta el naturalismo, rechaza el holismo quineano y se concentra solo en la práctica matemática sin tomar en cuenta nada del resto de la práctica científica. Esto es lo que ella llama la concepción de revisionismo interno de la matemática.

\section{Naturalismo matemático, el test de Maddy y cardinales grandes}

Ya que en otro lugar hemos analizado críticamente la propuesta revisionista de Maddy (Quezada 2005a y 2005b), la pasaremos ahora por alto y nos concentraremos en el test práctico que ella propone para desafiar la opinión de Quine. En el contexto de la teoría de conjuntos, como dijimos, Quine sostiene que el axioma de Gödel de constructividad $V=L$ es aquel que los matemáticos deberían preferir a la hora de reflexionar en sus pruebas sobre cardinales grandes (cf. Quine 1990, p. 95). Haciendo notar lo irónico de esta posición, Maddy muestra que ella se opone a la opinión de la mayoría de los actuales teóricos de conjuntos, pues, siguiendo el argumento de Quine, los matemáticos de acuerdo con $V=L$ deberían preferir un universo conjuntista austero, que preserve el axioma de elección y la hipótesis del continuo y contenga solo algunos cardinales grandes (por ejemplo, cardinales regulares e inaccesibles) ${ }^{4}$. Por otra parte, otros considerarán a $V=L$ una hipótesis que restringe de una manera inaceptable nuestro concepto general, iterativo, de un conjunto. Según ellos, las consecuencias inferenciales

2 Este axioma fue propuesto originalmente por Gödel en 1938. ' $V$ ' corresponde al universo de los conjuntos y ' $L$ ' a la clase de todos los conjuntos construibles de acuerdo a una condición recursiva (más adelante damos más detalles técnicos). Este axioma implica por tanto una restricción sobre los conjuntos aceptables, pues conjuntos no definibles de acuerdo a dicha condición no resultarán aceptables en la teoría estandard de conjuntos. A cambio, $V=L$ implica tanto la hipótesis del continuo como el axioma de elección y permite por tanto responder muchas cuestiones abiertas en la mencionada teoría. Sin embargo, como tendremos oportunidad de mostrarlo, este axioma genera más controversia que acuerdo entre los teóricos conjuntistas.

3 Un cardinal medible, en primera aproximación, es un cardinal más grande que un cardinal inaccesible con una medida no trivial K-completa con dos valores, definida sobre todos los subconjuntos de K. Así como los cardinales inaccesibles implican $V=L$, la existencia de cardinales medibles y en general no-construibles, implican, como probó Scott, su rechazo (cf. Scott 1961).

4 En opinión de Quine, $V=L$ "desactiva los vuelos más gratuitos de la teoría superior de conjuntos e incidentalmente implica el axioma de elección y la hipótesis del continuo" (Quine 1990, p. 95). 
más graves de dicho axioma serían, además de las severas restricciones internas sobre el universo conjuntista - por ejemplo, el rechazo del axioma de los cardinales medibles, del cardinal de Mahlo, etc.-, las externas: afectaría la anchura del buen orden de los reales, la existencia de los conjuntos medibles de acuerdo a una medida-no-Lebesgue, $\mathrm{y}$, en general, cualquier axioma que implique conjuntos no-construibles. Como señala Maddy, los teóricos de conjuntos y filósofos críticos de $V=L$ (entre los cuales el primero es el mismo Gödel (1947), en realidad, son claramente la mayoría (cf. Maddy 1997, pp. 84-85). Así, lo anterior sugiere que solo el rechazo al HN explicaría dos hechos ratificados por la práctica y la metodología internas de la teoría de conjuntos: (i) que en ZF (la teoría de conjuntos de Zermelo-Fraenkel) $V=L$ debería ser rechazado, y (ii) que, dado ese rechazo, debemos aceptar el axioma de existencia de los cardinales medibles (CM de aquí en adelante), y en general cardinales muy grandes inaccesibles y que no son construibles (cf. Kanamori 2004, Moschovakis 1994)5.

Por las anteriores razones, aunque nosotros concordamos plenamente con la posición de Maddy sobre $V=L$-apoyada en evidencia sustantiva y en la práctica efectiva de los matemáticos en torno a él- nos parece importante en este contexto defender que, si bien el naturalismo es la vía correcta para tratar las cuestiones fundamentales de la filosofía de la matemática, cuando ponemos el foco en la cognición matemática no necesitamos quedar presos ni del holismo quineano ni del revisionismo interno.

\section{Una respuesta naturalista alternativa: el enfoque cognitivista}

Como han argumentado varios autores, la posición de Maddy de rechazo a HN y a la tesis de indispensabilidad implica consecuencias epistémicas inaceptables, en particular un indeseable relativismo metodológico que no permitiría distinguir las matemáticas de, incluso, la astrología (cf. Pinto 2004, Dieterle 1999, Fernández de Castro 2005, Quezada 2005a). No volveremos sobre estas críticas generales aquí, sino que nos concentraremos en el caso de la teoría de conjuntos. Esto implica, a nuestro juicio, ofrecer una explicación alternativa a la de Maddy -pero en el marco de premisas naturalistas-de la práctica en teoría de conjuntos y, en particular, en el área de problemas sobre cardinales grandes. En particular, intentaremos mostrar que una respuesta naturalista más satisfactoria para el problema señalado se puede encontrar en trabajo desarrollado desde un área globalmente subestimada en filosofía de las matemáticas tradicional, la cognición matemática. Un trabajo semejante es el que ha sido elaborado por G. Lakoff y R. Núñez (2002), y que encuentra respaldo filosófico previo en Lakoff y Johnson (1999). Filosóficamente, estos autores rechazan cualquier empirismo o idealismo radical y se alinean con una visión que ellos tienden a catalogar como de mente corporeizada (embodied mind). Esta visión es naturalizada en el sentido preciso que la razón y los mecanismos cognitivos asociados a ella surgen de la naturaleza de nuestros cerebros, cuerpos y experiencia

$5 \quad$ Cf. en todo caso Devlin 1977 y 1979, para una defensa del axioma de constructividad. 
corporal, lo que subdetermina a su vez nuestra visión general de la razón. Es decir, la razón, de acuerdo a esta concepción, es evolutiva, situada (no universal en un sentido trascendente), mayormente inconsciente, y no puramente literal sino "extensamente metafórica e imaginativa” (Lakoff y Johnson 1999), p. 4). Esto significa, para nuestros propósitos aquí, asumir que el conocimiento matemático está íntimamente conectado con dichos fundamentos, lo que lo vuelve a su vez una extensión de nuestras actividades cognitivo-corporeizadas. En particular Lakoff y Núñez (L\&N de aquí en adelante) sugieren que los objetos matemáticos son conceptos corporeizados, esto es, ideas que se encuentran últimamente ancladas en la experiencia humana y que se conectan entre sí mediante mecanismos conceptuales humanos. Tales mecanismos fundamentalmente descansan en el uso de lo que ellos llaman metáforas conceptuales (conceptual metaphors) y mezclas conceptuales (conceptual blendingsf) ${ }^{6}$. En términos muy simplificados, los mecanismos aludidos se pueden visualizar como especificando relaciones (aplicaciones funcionales o mappings) entre dominios diferentes (normalmente llamados dominio fuente o dominio meta). Ejemplos simples son Estados son localizaciones y Categorías son contenedores. La primera metáfora mapea desde el dominio fuente ESPACIO al dominio meta ESTADO, la segunda desde el dominio fuente CONTENEDORES al dominio meta CATEGORÍAS (cf. Lakoff y Núñez op. cit., pp. 39-49).

La aritmética en general está, según L\&N, fundamentalmente basada en este tipo de metáforas. Sin embargo, su teoría se puede extender para cubrir otras áreas de la matemática. Por ejemplo, usando la metáfora Las Estructuras Algebraicas son Esencias Matemáticas podemos generar un mapeo desde los grupos conmutativos de 3 elementos en el dominio fuente a la adición módulo 3 en el dominio meta (que se entenderá como la esencia de la adición módulo 3) ${ }^{7}$. En este caso, la metáfora ya no es de anclaje sino de conexión, es decir, permite conceptualizar una rama de la matemática (álgebra de grupos) en términos de otra (aritmética modular). A su vez, estas metáforas son intensamente usadas en la conceptualización de la teoría de conjuntos. Por ejemplo, la metáfora Los Números Naturales son Conjuntos permite mapear conjuntos en el dominio fuente a números naturales en el dominio meta.

Un caso históricamente más interesante para nuestro argumento aquí es dado por la conceptualización de Cantor de los conjuntos infinitos, pues en ella mapeamos siempre entre dominios conjuntistas. Para comenzar, debemos admitir lo que L\&N llaman la metáfora básica de infinitud (MBI). MBI es una metáfora conceptual inconsciente que permite que procesos que transcurren indefinidamente sean conceptualizados como teniendo un fin y un resultado último. En este caso, el dominio meta son Procesos Iterativos Continuos o Sin Fin y el dominio fuente -que permite conceptualizar los primeros-son Procesos Iterativos Completos. Esto permite entonces que tales procesos sean concebidos como cosas u objetos infinitos, lo que está en la base de la noción cantoriana de infinito actual. La segunda metáfora cantoriana fundamental es, de acuerdo

6 Cf. para ambos conceptos Lakoff y Núñez 2002, pp. 39-45 y pp. 48-49, respectivamente.

7 Cf. ibíd., pp. 112-3. 
a lo que argumentan los autores, Mismidad numérica es Correspondencia Uno-a-Uno (es decir, biyección o pareabilidad) la que se puede graficar de la siguiente manera ${ }^{8}$ :

Dominio Fuente
Mapeos
Los conjuntos A y B
pueden ser puestos en
una correspondencia
uno-a-uno

Dominio Fuente

mapeo

$\rightarrow$

El conjunto A y el conjunto B tienen el mismo número de elementos

Lo valioso es que, según L\&N, estas conceptualizaciones metafóricas permiten explicar desde una perspectiva cognitiva cómo Cantor fue capaz de formular los conceptos de cardinal y ordinal transfinito. En el primer caso, podemos proponer el siguiente mapeo:

\section{Dominio Meta \\ PROCESOS ITERATIVOS SIN FIN \\ El estado de comienzo (0) \\ El estado de comienzo (0)}

Estado (1) que resulta de de la fase inicial del proceso El proceso a partir de un estado intermedio previo (n-1) produce el siguiente estado (n). El resultado intermedio después de la iteración del proceso.

El estado final resultante.

Implicación: el estado final resultante es único y sigue a todo estado final.

\section{Caso Especial del Dominio Meta $^{9}$ EL CONJUNTO DE LOS NUMEROS NATURALES}

$\rightarrow \quad$ Los números naturales y una operación de sucesor que forma un nuevo conjunto agregando 1 al último número

$\rightarrow \quad$ El conjunto vacío, cuya talla es el número cardinal 1.

$\rightarrow \quad$ Dado el cardinal $(\mathbf{1}, \ldots ., \mathbf{n}-\mathbf{1})$, forma el siguiente cardinal más grande $(\mathbf{1}, \ldots ., \mathbf{n})$.

$\rightarrow \quad$ El cardinal $(\mathbf{1}, \ldots ., \mathbf{n})$ cuya talla es el número cardinal $\mathrm{n}$.

$\rightarrow \quad$ El cardinal infinito $(\mathbf{1}, \ldots . \mathbf{n}, \ldots .$.$) cuya talla$ es el número cardinal transfinito $\mathrm{N} \omega$.

$\rightarrow \quad$ Implicación: $\omega$ es el único cardinal transfinito más pequeño y es más grande que cualquier número cardinal finito.

Ibíd., pp. 142-4.

Este caso especial, en realidad, es un caso especial de la misma MBI aplicado a los números naturales y a sus tallas. Sin embargo, se puede aplicar perfectamente a casos fuera de la misma matemática; cf., ibíd. pp. 161-2. 
Un mapeo semejante se podría dar también para explicar cómo, desde la perspectiva corporeizada, se generan los ordinales transfinitos, en cuyo caso el concepto de conjunto infinito es reemplazado por el de secuencia infinita ${ }^{10}$.

\section{Dominio Meta \\ PROCESO ITERATIVO SIN FIN NATURAL}

El estado de comienzo (0)

Estado (1) que resulta de de la fase inicial del proceso

El proceso a partir de un estado intermedio previo (n-1) produce el siguiente estado (n).

El resultado intermedio después de la iteración del proceso.

El estado final resultante.

Implicación: el estado final resultante es único y sigue a todo estado final.

\section{Caso Especial del Dominio Meta LA SECUENCIA DE LOS NUMEROS NATURALES EN SU ORDEN}

$\rightarrow \quad$ No hay secuencias

$\rightarrow \quad$ La secuencia de lugar-1 cuyo largo es el número ordinal 1.

$\rightarrow \quad$ Dada la secuencia $(\mathbf{1}, \ldots ., \mathbf{n}-\mathbf{1})$, forma la siguiente secuencia más larga $(1, \ldots ., n)$

La secuencia $(\mathbf{1}, \ldots ., \mathbf{n})$ cuyo largo es el número ordinal transfinito $\mathrm{n}$.

La secuencia infinita $(\mathbf{1}, \ldots \mathbf{n}, \ldots$.$) cuyo$ largo es el número cardinal transfinito ro. Implicación: ro es el único ordinal transfinito más pequeño y es más largo que cualquier número ordinal finito.

Dadas las limitaciones de espacio, no es éste el lugar para explicar adicionalmente los anteriores mecanismos y cómo operan en las prácticas matemáticas concretas. En su lugar, como dijimos, nos concentraremos en ofrecer razones adicionales para admitir la importancia de proveer una fundamentación cognitivo-naturalista, del tipo corporeizado, a la idea o concepto de cardinal medible y, por tanto, no-construible, que se seguiría del rechazo de $V=L$.

\section{Una caracterización corporeizada de conjuntos construibles y no construibles}

Ya que en una publicación anterior (Quezada 2007) hemos sugerido cómo dar sentido a la propuesta de base cognitivista para caracterizar un conjunto construible, ahora solo resumiremos los pasos básicos de la estrategia, para luego aclarar cómo se puede modelar cognitivamente la caracterización de los conjuntos no-construibles. 
Para hacerse una idea básica de lo que sea un conjunto construible es fundamental ofrecer una idea de la jerarquía acumulativa de conjuntos a partir de la cual, como sugirieron von Neumann y Zermelo, se puede obtener el universo conjuntista $(V)$. En dicha jerarquía, $V$ se concibe como el resultado de un proceso recursivo que, partiendo del conjunto vacío, genera los conjuntos a partir de otros ya obtenidos anteriormente mediante la aplicación de las operaciones de conjunto potencia $(\wp)$ y gran unión (U). Cada escalón de la jerarquía se puede ver entonces como la indexación mediante un ordinal $\alpha$. Esto se puede visualizar de la siguiente clásica manera.

$$
\begin{aligned}
& V(0)=\varnothing \\
& (\alpha+1)=\wp V(\alpha)(\text { para cualquier ordinal } \alpha) \\
& \left.\mathrm{V}(\lambda)=\sup \{V(\beta): \beta<\lambda\}=\mathrm{U}_{\beta<\lambda} V(\beta) \text { (para cualquier ordinal límite } \lambda\right)
\end{aligned}
$$

La gran unión de todos los escalones, que podemos representar como $\mathrm{U}_{\alpha \in \Omega} V(\alpha)$, es entonces la jerarquía acumulativa ${ }^{11}$. La postulación de $V=L$ significa así identificar esta unión con la clase de los conjuntos construibles, es decir, significa admitir que los conjuntos que se pueden obtener mediante la jerarquía acumulativa solo pueden pertenecer a dicha clase. Esto sugiere que la jerarquía misma no resulta suficiente para caracterizar la noción de conjunto arbitrario y que para ello se debe agregar una restricción. Sin esta restricción podríamos aceptar una concepción de la jerarquía, y por tanto del universo de conjuntos, más liberal o en cualquier caso caracterizar los conjuntos de acuerdo a otra restricción.

Debemos a continuación clarificar en qué consiste un conjunto construible. Para ello, me concentraré aquí en la versión más semántica ofrecida por Gödel (1938 y 1939). En términos muy simplificados, Gödel determina lo que es un conjunto construible mediante un modelo $\mathrm{M}$ donde todo conjunto es obtenible mediante la jerarquía ramificada de los tipos de Russell extendida a órdenes transfinitos y con la restricción de que la colección de los subconjuntos del orden $\alpha$, a partir de la cual se genera el conjunto potencia, sea definible por fórmulas de primer orden que no impliquen impredicatividad $^{12}$. Por otro lado, la restricción a procedimientos no-impredicativos, aunque parece sugerir que $\mathrm{M}$ no permite en particular definiciones impredicativas de

11 Uno podría visualizar la jerarquía como un cono invertido cuyo eje es dado por la serie de los ordinales y que puede modificarse tanto en altura como en anchura, dependiendo de la aceptación de determinados axiomas que exceden a ZF (o cualquier otra axiomatización clásica). Por ejemplo, la aceptación del axioma de constructibilidad o la hipótesis del continuo determinarán la anchura, la aceptación de axiomas de cardinales grandes o supergrandes determinarán la altura. Desde luego, opciones en cada lado tendrán consecuencias en el otro lado.

12 Cf. Gödel 1938, p. 211. Ya que la alusión a la teoría de los tipos de Russell no es fundamental en una definición contemporánea de la jerarquía acumulativa podemos desatenderla, cf. Devlin 1979, cap. V. 
conjuntos $^{13}$, tiene como propósito general sugerir que los conjuntos aceptables en él deben caracterizarse constructivamente, es decir, mediante una definición explícita. Pero la única forma de obtener una caracterización constructiva adecuada de conjunto (arbitrario) en la jerarquía acumulativa transfinita es que sepamos definir la noción de conjunto de las partes de un conjunto infinito. Y esto nos lleva necesariamente a la segunda línea de la caracterización de la jerarquía dada arriba, es decir, a la aplicación de la operación de conjunto potencia. De acuerdo a Gödel (1939 y 1940), y en términos simples, dado un conjunto infinito A y un subconjunto $\mathrm{x}$ de él podemos decir que $\mathrm{x}$ es un subconjunto definible de A si y solo si existe una fórmula $\varphi(x)$ del lenguaje de primer orden de la teoría de conjuntos con la restricción fundamental de que existan las suficientes constantes o parámetros para cada elemento de $\mathrm{A}$ (o de $\mathrm{M}$ dado el ordinal $\alpha$ respectivo). De manera que $\mathrm{x}$ es un subconjunto definible en A si y solo si $\{\mathrm{x} \in \mathrm{A}: \varphi(\mathrm{x})\}$. Podemos entonces llamar $\mathrm{Df}(\mathrm{A})$ al conjunto de todos los subconjuntos de A definibles en A y reemplazar consecuentemente por este símbolo la operación de conjunto potencia en la segunda línea mencionada ${ }^{14}$. Si además reemplazamos $V$ (es decir, "construible") por $L$ en todas las líneas, obtenemos la siguiente caracterización de los conjuntos construibles y por ende de la jerarquía acumulativa.

$$
\begin{aligned}
& L(0)=\varnothing \\
& (\alpha+1)=\operatorname{Df} L(\alpha) \text { (para cualquier ordinal } \alpha) \\
& \left.L(\lambda)=\sup \{L(\beta): \beta<\lambda\}=\mathrm{U}_{\beta<\lambda} L(\beta) \text { (para cualquier ordinal límite } \lambda\right)
\end{aligned}
$$

Simplificando aún más y relativizando la caracterización al modelo M de Gödel (1939), podemos decir que $\mathrm{x}$ es construible en una jerarquía ordinal si y solo si existe un número ordinal $\alpha$ tal que $\mathrm{x} \in \mathrm{M}_{\alpha}$ De esta manera, resulta claro que la caracterización de un conjunto construible descansa en una operación de definibilidad que permite asignar a cada subconjunto de un conjunto de la jerarquía una fórmula dentro de un lenguaje de primer orden, no importando de qué ordinal se trate, e incluyendo por tanto al conjunto de los subconjuntos.

La anterior caracterización resulta en principio suficiente para los propósitos de formular una base cognitivista mínima de los conjuntos construibles. Para ello, ya que la concepción recursiva de los conjuntos de von Neumann es extensional y presupone intuitivamente la inclusión de objetos (del tipo que sea), podemos con L\&N aplicar, entre otras, las metáforas conceptuales de conjuntos son contenedores ${ }^{15}$ y conjuntos

13 Una definición impredicativa de un conjunto es aquella que alude a una totalidad de los conjuntos a la cual pertenece también el conjunto definido.

14 Obviamente, aunque $\operatorname{Df}(\mathrm{A}) \mathrm{c} \mathrm{pA}$, no se sostiene la conversa.

15 Cf. Lakoff y Núñez 2002, pp. 31 y ss. respecto a Esquemas de contenedor y pp. 140-1 sobre esta metáfora y su relación con la operación de conjunto potencia. 
son objetos construidos ${ }^{16}$. Por otro lado, dado que muchos cardinales grandes, por ejemplo, algunos cardinales inaccesibles, son conjuntos construibles también, la generación cognitiva de la jerarquía acumulativa implica aplicar MBI. Sin embargo, ya que los conjuntos construibles imponen límites a la colección de subconjuntos del orden a, MBI debe poner restricciones a los procesos indefinidos, es decir, no cualquier iteración puede originar un conjunto construible ${ }^{17}$. La cuestión crucial entonces es caracterizar dicha restricción. La clave se debe encontrar, total o parcialmente, en la caracterización de la noción de subconjunto (o parte) de un conjunto infinito. Como hemos visto, ésta es una noción dependiente-de-lenguaje, en particular, dependiente de un lenguaje de primer orden.

Bajo esta concepción, sugiero, por tanto, que podemos aislar claras metáforas conceptuales de este tipo para la noción de subconjunto de Gödel. La primera es que subconjuntos son conjuntos de fórmulas y la segunda es que todo conjunto es especificable mediante una fórmula en un lenguaje (véase Gödel 1939: 215 y s.). La operación de subconjunto (o de conjunto potencia de un conjunto construible) es por tanto una operación lingüística que consiste en dar un nombre a cada ordinal a partir del cual se forma el conjunto de los subconjuntos (los cuales también deberán contar de nombres). De modo que, de acuerdo a nuestra explicación anterior sobre la definibilidad de ordinales de Gödel, somos llevados a pensar que en la generación de la jerarquía acumulativa todo matemático constructivista tendrá que admitir que especificar el conjunto de las partes de un conjunto es asignar un nombre (mediante una fórmula) a dicho conjunto en una serie ordinal. En otras palabras, el mapping que conecta subconjuntos (en el dominio meta) con nombres de ordinales (en el dominio fuente) es una relación de bautismo. Por tanto, en términos de metáforas conceptuales, parece razonable sugerir algo como lo siguiente como un buen candidato para capturar dicho proceso: Determinar los subconjuntos de un conjunto dado es bautizar un conjunto en un cierto orden.

Reunimos aquí las metáforas en las que, en nuestra opinión, descansa, al menos parcialmente, la concepción de los conjuntos construibles desde un punto de vista cognitivo-corporeizado.

1. Metáforas de anclaje: conjuntos son contenedores conjuntos son objetos construidos

2. Mezcla conceptual: metáfora básica de infinitud (con restricción)

16 Para esta metáfora, cf., ibíd., pp. 140-146. Ambas metáforas son metáforas de anclaje, que proporcionan ideas directamente fundadas en experiencia sensible.

17 Esto restringirá también la clausura generativa de las operaciones de conjunto potencia y unión; cf., ibíd., p. 176. 


\section{Restricción de $M B I$}

3. Metáforas de subconjunto: subconjuntos son conjuntos de fórmulas / Todo conjunto es especificable mediante una fórmula en un lenguaje formal

4. Metáfora de conjunto potencia: determinar los subconjuntos de un conjunto dado es bautizar el conjunto de los subconjuntos en una serie ordinal.

Lo anterior permite sugerir entonces un camino inicial para caracterizar, desde una perspectiva cognitiva, y de una manera conversa, el concepto de conjunto no-construible. La clave es que, de acuerdo a lo que sugieren $L \& N$ sobre formación del conjunto potencia de cardinales transfinitos, implicará una operación que debe ajustarse a la aplicación de MBI (mediante la restricción propuesta más arriba) y cuyo resultado se asociará a un ordinal de la jerarquía acumulativa, una operación que debería ajustarse perfectamente al procedimiento de definibilidad de ordinales propuesto por Gödel. En palabras de L\&N:

A power set is always larger than the set it was based on. Moreover, given a power set, we can always form the power set of that power set, and so on. This is true for infinite as well as finite sets within Cantor's set theory. Given a set of cardinality No we can form the power set of the power set of ... of the power set of the natural numbers. This is a hierarchy of sets of everincreasing Cantor size [.... ] To summarize, the Basic Metaphor of Infinity allows us to form the entire set of natural numbers. Cantor's metaphor, applied with the BMI, allows us to derive the cardinal number, No, for the entire infinite set of natural numbers. Cantor's metaphor and the BMI jointly allow us to "prove" that there are "more" reals than natural numbers and rational numbers. Cantor's metaphor allowed Cantor to prove that there are always more elements in a power set than in its base set, even for infinite sets. And this result provides a hierarchy of transfinite cardinal numbers in Cantor's set theory (Lakoff y Núñez 2002, pp. 215-6).

Conversamente entonces debemos esperar que para obtener conjuntos no-construibles debemos modificar o simplemente abandonar algunas de las restricciones propuestas arriba sobre MBI. Esto desde luego implica algo impensado pero, tal vez, inevitable en una concepción no-constructivista de los cardinales grandes: que no todo conjunto (por ejemplo, los conjuntos de todos los subconjuntos de un conjunto dado) puede ser definido por un procedimiento de nominación unívoco. Desde el punto de vista cognitivo, esto significa que, si no renunciamos a la metáfora de Cantor de pareabilidad y a MBI, entonces la pareabilidad de conjuntos y la ordenación en una jerarquía transfinita no implica necesariamente identificación nominal de los elementos pareados y ordenados.

Por otra parte, desde el punto de vista conjuntista, esta consecuencia tal vez permitiría indicar una hipótesis explicativa productiva acerca de por qué normalmente algunos cardinales muy grandes (cardinales medibles, fuertes, de Woodin, supercompactos, etc.) parecen no poder ser discriminados o ser discernibles, dentro de una estructura 
$(L, \epsilon)^{18}$. Esta indiscernibilidad podría perfectamente entenderse como una consecuencia de admitir que no todos los ordinales respectivos necesitan ser identificados mediante un nombre en un lenguaje formal dado, es decir, bautizados en él ${ }^{19}$. Si esta hipótesis tiene algún sustento, entonces proporciona un indicio para entender qué es lo que los matemáticos trabajando con conjuntos no-construibles -la mayoría, como indica Maddyconceptualizan cuando prueban teoremas o propiedades acerca de tales conjuntos.

Finalmente, lo anterior puede sugerir algunas líneas sobre cómo interpretar cognitivamente los resultados de P. Cohen sobre la independencia de la Hipótesis del Continuo que mencionamos anteriormente. En relación con esto, L\&N solo afirman lo siguiente:

Cantor stated the Continuum hypothesis as a conjecture, which he was never able to prove. Later, Kurt Gödel (in 1938) and Paul Cohen (in 1963) proved theorems that together showed that the conjecture is neither absolutely true nor absolutely false relative to generally accepted axioms for set theory. Its truth depends on what further axioms one takes "set" to be defined by. From the perspective of our mathematical idea analysis, this means that whether or not the Continuum hypothesis is "true" depends on the underlying conceptual metaphors characterizing the concept "set" (Lakoff y Núñez 2002, p. 215).

Sin entrar en mayores detalles aquí sobre la prueba de Cohen, podemos decir que apeló a métodos modelo-teoréticos que le permitieron extender el universo de los conjuntos $\mathrm{V}$ especificando una extensión genérica $\mathrm{G}$ de él que se adjunta luego a un modelo -el modelo $\mathrm{M}(\mathrm{G})$ - de ZF donde no es válida HC (ni tampoco el Axioma de Elección). Para ello, Cohen propuso una técnica semántica original, la técnica del forcing, asociada a un lenguaje en particular (Cohen 1966, Moore 1988). El lenguaje en el que se aplica la relación de forcing -una condición p fuerza una fórmula $\varphi$ del lenguaje a ser verdadera- presupone un conjunto en el cual a todas las condiciones que fuerzan se les asigna por inducción transfinita un nombre (los P-nombres), lo que permite sustituir luego el nombre por su valor, asignar valuaciones a las fórmulas del lenguaje de forcing y, con ello, especificar su definibilidad en la extensión genérica.

18 Esta sugerencia se basa en el descubrimiento de los números reales $0^{\#}$ (zero sharps) por Silver, los que muestran que una diferencia importante entre $V$ y $L$ parece residir en la indiscernibilidad de los cardinales incontables en $L$. La existencia de cardinales medibles y otros cardinales más grandes presupone la existencia de sharps (véase Maddy 1997: cap. 5).

19 Gödel mismo (en Gödel 1946) ha sugerido su propia explicación acerca de cómo $V$ podría discrepar de $L$. Según Gödel, la noción de definibilidad de los ordinales en las cuales se basa su caracterización de los conjuntos construibles es una formulación adecuada y absoluta de la propiedad "estar formados según una ley” y en cambio la no-definibilidad (y la no-constructibilidad) reflejaría la propiedad "estar formados por una elección aleatoria de elementos" (Gödel 1946: 349). Aunque pienso que no es imposible hacer compatible mi explicación cognitivo-corporeizada de los conjuntos construibles con la de Gödel, no entraré en esta materia aquí. 
Esto en principio basta para probar la falsedad de $\mathrm{HC}$ en $\mathrm{M}(\mathrm{G})$. Nos gustaría sugerir aquí que para una reconstrucción cognitivista de este método se hace necesario, en nuestra opinión, reinterpretar la construcción del lenguaje de forcing apelando a algunas de las metáforas que hemos utilizado más arriba para definir conjuntos construibles, en particular, la metáfora de subconjunto y la metáfora de conjunto potencia, las que especifican una relación de bautismo para los elementos de un conjunto dado en una serie ordinal. Esto podría, tal vez, revelar más de lo que L\&N sugieren en la cita precedente, esto es, que los presupuestos conjuntistas de la prueba de Cohen resultarán muy distantes de los cantorianos pues el conjunto genérico representará un tipo de infinito no-cantoriano. El desafío obvio entonces es testar esta hipótesis de acuerdo a las líneas cognitivo naturalistas propuestas por $\mathrm{L} \& \mathrm{~N}$ y especificar los elementos de base cognitivista restantes que la hagan suficientemente explicativa.

\section{Referencias bibliográficas}

Alemán, A. (2001), Lógica, matemáticas y realidad. Madrid: Tecnos.

Benacerraf, P. (1973), "Mathematical Truth", en Hart (1976).

Cohen, P., (1966), Set Theory and the Continuum Hypothesis. New York: Benjamín.

Colyvan, M. (2001), The indispensability of mathematics. Oxford: Oxford University Press.

Devlin, K. (1977), The axiom of constructibility. Berlin: Springer Verlag. Verlag.

(1979), Fundamentals of Contemporary Set Theory. New York: Springer

Dieterle, J. M. (1999), "Mathematical, Astrological, and Theological Naturalism", Philosophia Matemática (3), vol. 7: 129-135.

Drake, F. (1974), Set Theory: An Introduction to Large Cardinals. Amsterdam: North Holland.

Fernández, M. (2005), "El revisionismo en filosofía de la matemática”, Signos Filosóficos, vol. VII, N 13: 115-120.

Field, H. (1980), Science without Numbers: a defense of nominalism. Oxford: Basil Blackwell.

(1989), Realism, Mathematics and Modality, Oxford: Blackwell.

Gödel, K. (1938), "La consistencia del axioma de elección y de la hipótesis generalizada del continuo", en K. Gödel (1989).

(1939), "Prueba de consistencia de la hipótesis generalizada del continuo", en K. Gödel (1989).

(1940), "La consistencia del axioma de elección y de la hipótesis generalizada del continuo con los axiomas de la teoría de conjuntos", en K. Gödel (1989). 
(1947) “Cuál es el problema del continuo?” en Gödel, K., Obras Completas ( $2^{\mathrm{a}}$ ed.) traducción y comentarios de J. Mosterín, Alianza Editorial, 1989.

(1989), Obras Completas (2a ed.), traducción y comentarios de J. Mosterín. Madrid: Alianza Editorial.

Hart, W. D. (1996), (ed.) The Philosophy of Mathematics. Oxford: Oxford University Press.

Kanamori, A. (2004), 2a ed. The Higher Infinite. N. York: Springer Verlag.

Kitcher, P. (1984), The Nature of Mathematical Knowledge. Oxford: Oxford University Press.

Kunen K. (1980), Set Theory. An Introduction to Independence Proofs. Amsterdam: North-Holland.

Lakoff, G. y M. Johnson (1999), Philosophy in the Flesh: The EmbodiedMind and its Challenge to Western Thought. New York: Basic Books.

Lakoff, G. y R. Núñez (2002), Where Mathematics Come From: How the Embodied Mind Brings Mathematics Into Being. New York: Basic Books.

Maddy, P. (1990), Realism in Mathematics. Oxford: Clarendon Press.

(1997), Naturalism in Mathematics. New York: Oxford University Press.

Moore, G. (1988), “The origins of forcing”, en F. Drake y J. Truss (eds.), Logica Colloquium 86. Amsterdam: North -Holland.

Moschovakis, Y. (1994), Notes on Set Theory. N. York: Springer Verlag.

Pinto, S. (2004), "Revisionismo, anti-revisionismo y filosofía de las matemáticas", Signos Filosóficos $\mathrm{N}^{\circ} 12$.

Putnam, H. (1975), "What is mathematical truth?”, en Putnam (1979).

(1979), Mathematics, Matter and Method, vol. I. Cambridge: Cambridge University Press.

Quine, W. (1951), “Two dogmas of empiricism”, en Quine (1980).

(1955), "Posits and reality", en Quine (1976).

(1969), "Epistemology naturalized", en Quine (1969).

(1969), Ontological relativity and other Essays. New York: Columbia University Press.

(1976), The Ways of Paradox and Other essays. Cambridge Mass.: Harvard University Press.

(1980), From a Logical Point of View. Cambridge Mass.: Harvard University Press. Press.

(1981), Theories and Things. Cambridge Mass.: Harvard University

(1990), Pursuit of Truth. Cambridge Mass.: Harvard University Press.

Quezada, W. (2004a), "Ficcionalismo y la filosofía de la matemática de Russell". Ponencia presentada al IV Encuentro de la Afhic. Buenos Aires. 
(2004b), "Ficcionalismo matemático y si-entoncismo russelliano ¿dos caras de la misma moneda?", Revista de Filosofia, Universidad Complutense de Madrid, vol. 29, $\mathrm{N}^{\circ}$ 2: 73-97.

(2005a), "Revisionismo y Naturalismo en Filosofía de las Matemáticas". Ponencia presentada al XIII Congreso Nacional de Filosofía de la AFRA. Rosario, Argentina.

(2005b), “QQuién revisa, cómo se revisa y qué hace posible revisar la matemática?", Signos Filosóficos. México, vol. VII, N 13: 107-114.

(2007), "Revisionismo y cognitivismo en filosofía de la matemática", en Selección de Trabajos VII Jornadas Rolando Chuaqui en Filosofía y Matemáticas. A. Bobenrieth (ed.), Edeval, Valparaíso, 2007, pp. 47-57.

Resnik, M. (1983), "Review of Field (1980)", Nous, 27, 514-19.

(1997), Mathematics as a science of patterns. Oxford: Oxford University Press.

Russell, B. (1903), The Principles of Mathematics. Norton \& Co., New York (hay trad. en español: Los Principios de la Matemática. Bs. Aires: Espasa-Calpe, 1948).

(1973), Essays in Analysis. D. Lackey (ed.). London: G. Allen \& Unwin.

Scott, D. (1961), "Measurable cardinals and constructible sets”, Bull. Acad. Pol. Sci., 9: $521-4$.

Shapiro, S. (1983), "Conservativeness and incompleteness", Journal of Philosophy 80: $521-31$.

(1997), Philosophy of Mathematics: Structure and Ontology.

Tennant, N. (2000), "What is Naturalism in Mathematics, Really?", Philosophia Mathematica (3), vol. 8: 316-338.

Torretti, R. (1998), El paraíso de Cantor. La tradición conjuntista en filosofía matemática. Santiago: Editorial Universitaria.

Torretti, R. y J. Mosterín (2002), Diccionario de Lógica y Filosofía de la Ciencia. Madrid: Alianza.

Wagner, S. (1982), “Arithmetical Fiction”, Pacific Philosophical Quarterly 63: 25569. 